\title{
PARADOXICAL PROPOSITIONS
}

\author{
Graham Priest
}

July 2, 2018

\begin{abstract}
Departments of Philosophy, the CUNY Graduate Center and the University of Melbourne
\end{abstract}

\begin{abstract}
This paper concerns two paradoxes involving propositions. The first is Russell's paradox from Appendix B of The Principles of Mathematics, a version of which was later given by Myhill. The second is a paradox in the framework of possible worlds, given by Kaplan. This paper shows a number of things about these paradoxes. First, we will see that, though the Russell/Myhill paradox and the Kaplan paradox might appear somewhat different, they are really just variants of the same phenomenon. Though they do this in different ways, the core of each paradox is to use the notion of a proposition to construct a function, $f$, from the power set of some set into the set itself. Next we will see how this paradox fits into the Inclosure Schema. Finally, I will provide a model of the paradox in question, showing its results to be non-trivial, though inconsistent.
\end{abstract}

\section{Introduction}

There are a number of paradoxes of self-reference which are intensional, in that they involve propositions - intensional entities of a certain kind. ${ }^{1}$ This paper concerns two such. Both are well known.

\footnotetext{
${ }^{1}$ For a discussion of a number of such paradoxes, see Priest (1991).
}

This is the author manuscript accepted for publication and has undergone full peer review but has not been through the copyediting, typesetting, pagination and proofreading process, which may lead to differences between this version and the Version of Record. Please cite this article as doi: 10.1111/phis.12126

This article is protected by copyright. All rights reserved. 
The first is due to Russell in Appendix B of The Principles of Mathematics. $^{2}$ It is also rehearsed by Myhill. ${ }^{3}$ The paradox concerns the set of propositions, $P$. It shows how, for any subset, $X$, of $P$, to construct a proposition, $p_{X}$, in such a way that distinct subsets generate distinct propositions. But this is impossible, since there are more subsets of propositions than propositions.

The second paradox is due to Kaplan. ${ }^{4}$ Kaplan is working in a worldsframework, which takes propositions to be subsets of the set of worlds, $W .^{5}$ For any proposition, that is, subset of $W, X$, Kaplan shows, how to identify a world, $w_{X}$, in such a way that different subsets deliver different worlds. But this is impossible, since there are more subsets of worlds than worlds.

In this essay, I will show a number of things. First, we will see that, though the Russell/Myhill paradox and the Kaplan paradox might appear somewhat different, they are really just variants of the same phenomenon. Though they do this in different ways, the core of each paradox is to use the notion of a proposition to construct a function, $f$, from the power set of some set into the set itself. ${ }^{6}$ Next we will see how this paradox fits into a very familiar schema: the Inclosure Schema. Finally, I will provide a model of the paradox in question, showing its results to be non-trivial, though inconsistent.

\section{Cantor's Paradox}

As is clear from what I have just said, the two versions of our paradox are intensional variants of Cantor's Paradox. So, to set things up, let us start with a proof of this.

First, Cantor's Theorem: there is no function from the powerset of a set into the set. Proof:

Let $S$ be any set, and $\wp(S)$ its power-set. Suppose that there is a function, $f$, from $\wp(S)$ into $S$. Define a subset, $R$, of $S$, as follows:

\footnotetext{
${ }^{2}$ Russell (1903), sec. 500.

${ }^{3}$ Myhill (1958).

${ }^{4}$ Kaplan (1995).

${ }^{5}$ If one wishes, one can take worlds themselves to be propositions: "maximally consistent" propositions.

${ }^{6}$ To remind: a function, $f$, is into if it maps distinct objects to distinct objects. That is, if $x \neq y$ then $f(x) \neq f(y)$.
} 
- $R=\{x \in S: \exists Y(x=f(Y) \wedge \neg x \in Y)\}$

Since, $R \subseteq S$, for some $w \in S, w=f(R)$. Now suppose that:

- $w \in R$

Then:

- $\exists Y(w=f(Y) \wedge \neg w \in Y)$ (Set Abstraction)

Instantiating:

- $w=f(C) \wedge \neg w \in C$

But since $f$ is into, $w=f(R)$ and $w=f(C)$ entail that $R=C$. Hence:

- $\neg w \in R$

But then:

- $w=f(R) \wedge \neg w \in R$

So:

- $\exists Y(w=f(Y) \wedge \neg w \in Y)$

and so:

- $w \in R$ (Set Abstraction)

Consequently, there is no such $f$.

But of course, if there is such an $f$, as there would appear to be if $S$ is the set of all sets (where $f$ may be the identity function), we have a contradiction. This is Cantor's Paradox.

\section{Propositional Paradoxes}

Let us now see how our intentional paradoxes deploy this framework.

In the Russell/Myhill paradox, $S$ is the set of propositions, $P$, and if $Y \subseteq S$, then, using the notation of propositional quantification, $f(Y)=$ $(\forall p \in Y) p . f$ is into, since if $X \neq Y$ then $(\forall p \in Y) p$ and $(\forall p \in X) p$ are distinct propositions. So $f(X) \neq f(Y)$. This is the Russell construction. In the Myhill construction $f(Y)=\forall p p \in Y$. The result is the same. 
In Kaplan's paradox, $S$ is the set of worlds, and propositions are subsets of worlds, so $P$ is $\wp(S)$, the set of propositions. If $Y \subseteq S$ then $f(Y)=$ $\varepsilon w\left(\operatorname{Ext}_{w}(F)=\{Y\}\right)$, where $F$ is some fixed monadic non-logical predicate of propositions, $\operatorname{Ext}_{w}(F)$ is the extension of $F$ at $w, \varepsilon w$ selects some world satisfying the condition which follows it. We make the natural assumption that for any proposition, $Y$, there are worlds where the extension of $F$ is just $\{Y\} . f$ is into, since if $X \neq Y$ then $\varepsilon w\left(\operatorname{Ext}_{w}(F)=\{X\}\right) \neq \varepsilon w\left(\operatorname{Ext}_{w}(F)=\right.$ $\{Y\})$. So $f(X) \neq f(Y)$.

As is clear, each argument uses the machinery of propositions to construct a map, $f$, from the power set of some set into the set itself. Details of the construction depend on various different assumptions about propositions; but these are just means for achieving the same end - just as different means may be used to obtain the self-reference required for the liar paradox (descriptions, demonstratives, Gödel coding).

\section{The Inclosure Scheme}

Let us now see how the paradox fits the Inclosure Schema. This is a general schema into which all the standard paradoxes of self-reference fit. ${ }^{7}$ The Schema concerns a totality, $\Omega$, a monadic predicate, $\psi$, and a function, $\delta$, such that:

- $\Omega$ exists and $\psi(\Omega)$ (Existence)

- If $X \subseteq \Omega$ is such that $\psi(X)$ :

$$
\begin{aligned}
& -\delta(X) \notin X \text { (Transcendence) } \\
& -\delta(X) \in \Omega \text { (Closure) }
\end{aligned}
$$

Applying Transcendence and Closure to $\Omega$ itself, delivers the contradiction that $\delta(\Omega) \in \Omega$ and $\delta(\Omega) \notin \Omega$. There is a special case of the Schema when $\psi$ is the vacuous condition, $X=X$, and so may be ignored. This is the Russell Schema.

\footnotetext{
${ }^{7}$ See Priest (2002a), Part 3. Perhaps the major controversy over this to date concerns the question of whether Curry's paradox does, or ought to, fit the Schema. This is not the place to discuss that matter. Further on this matter, see $\S 17$ of Priest (2017).
} 
Our propositional paradox fits the Russell Schema. The argument for this is little more than the corresponding argument to show that Cantor's Paradox fits the Schema.

Take $\Omega$ to be to range of our lower case variables (so that upper case variables range over subsets of this). And let $\delta(X)$ be $f\left(X^{\prime}\right)$, where $X^{\prime}=$ $\{x \in X: \exists Y(x=f(Y) \wedge \neg x \in Y)\}$.

The paradox assumes Existence (a totality of all propositions or worlds). Closure holds, since $f$ is a map into $\Omega$. It remains to check Transcendence. The argument for this is simply a version of our paradoxical argument. Suppose that $X \subseteq \Omega$. Let $w=\delta(X)=f\left(X^{\prime}\right)$. We then argue as follows.

First, suppose that $w \in X^{\prime}$. Then:

- $\exists Y(w=f(Y) \wedge w \notin Y)$

Instantiating:

- $w=f(C) \wedge w \notin C$

But since $f$ is into, $w=f\left(X^{\prime}\right)$ and $w=f(C)$ entails that $X^{\prime}=C$. Hence:

- $w \notin X^{\prime}$

Hence, by Excluded Middle: $w \notin X^{\prime}$. But then:

- $w=f\left(X^{\prime}\right) \wedge w \notin X^{\prime}$

So:

- $\exists Y(w=f(Y) \wedge w \notin Y)$

Given the definition of $X^{\prime}$, if $w \in X$ then $w \in X^{\prime}$, which it is not. So $w \notin X$, as required.

\section{$5 \quad$ Properties for Sets}

This is not the place to discuss possible suggestions for solving our target paradox. Some of these are discussed in the references already cited. But given that it is an Inclosure Paradox, and I have advocated a dialetheic solution for such paradoxes, ${ }^{8}$ I want in what follows to investigate further

\footnotetext{
${ }^{8}$ E.g., Priest (2002a).
} 
such a solution. The aim will be to find a model of the paradox which shows that the paradoxical argument does not deliver triviality. We will do this by a little massaging (interesting, I think, in its own right), which will come in two stages.

First, note that the identity conditions of sets are playing no role in the argument. We might just as well, then, take upper case variables to range over properties, replace set abstracts with $\lambda$-terms, and set membership with instantiation, $\eta$. The principle of set abstraction transforms into $\lambda$ conversion. The paradoxical argument then becomes:

- $R=\lambda x(\exists Y(x=f(Y) \wedge \neg x \eta Y)$

- $w=f(R)$

Suppose that:

- $w \eta R$

Then:

- $\exists Y(w=f(Y) \wedge \neg w \eta Y)(\lambda$-conversion $)$

Instantiating:

- $w=f(C) \wedge \neg w \eta C$

But since $w=f(R)$ and $w=f(C)$ then $R=C$. Hence:

- $\neg w \eta R$

Also:

- $w=f(R) \wedge \neg w \eta R$

So:

- $\exists Y(w=f(Y) \wedge \neg w \eta Y)$

and so:

- $w \eta R(\lambda$-conversion $)$ 


\section{Open Sentences for Properties}

Next, the argument assumes nothing about the identity conditions for properties either. We may therefore simply take them to be open sentences, with at most one free variable, $y$. $\lambda$-terms are replaced by the names of sentences. So if $A$ is any such sentence, let $\langle A\rangle$ be its name. Instantiation may then be replaced by the satisfaction relation, $\sigma$. So $x \sigma Y$ means that $x$ satisfies the sentence with one free variable, $Y . \lambda$-conversion morphs into the Satisfaction Scheme:

$$
\text { - } t \sigma\langle A\rangle \text { iff } A_{y}(t)
$$

where $t$ is any term, and $A_{y}(t)$ is $A$ with occurrences of $y$ replaced by $t$ (with relabelling of bound variables if necessary, to avoid clashes).

Note that $A$ may have no free variables. And if we are taking open sentences for properties, we can take closed sentences to be propositions. Fix some term, $t$. If $A$ has no free variables, the Satisfaction Schema then becomes:

- $t \sigma\langle A\rangle$ iff $A$

So ' $t \sigma$ ' is a truth predicate.

Our argument now becomes the following. ${ }^{9}$

- $R=\langle\exists Y(v=f(Y) \wedge \neg v \sigma Y)\rangle$

- $w=f(R)$

Suppose that:

- $w \sigma R$

- then $\exists Y(w=f(Y) \wedge \neg w \sigma Y)$ (Satisfaction Schema)

Instantiating:

- $w=f(C) \wedge \neg w \sigma C$

Since $w=f(R)$ and $w=f(C)$ then $R=C$. Hence:

\footnotetext{
${ }^{9}$ Note that we assume here that $f$ is a definable function. What is inside the quotation is, strictly speaking, its definition.
} 
- $\neg w \sigma R$

Also:

- $w=f(R) \wedge \neg w \sigma R$

But then:

- $\exists Y(w=f(Y) \wedge \neg w \sigma Y)$

and so:

- $w \sigma R$ (Satisfaction Schema)

\section{A Model for the Paradoxes}

We are now in a position to produce a formal model for our paradox. We take the language to be that of first-order arithmetic, augmented by a binary predicate, $\sigma$. Gödel coding, \#, is used to deliver the names for formulas. Lower case variable are taken to range over numbers; and upper case variables range over (codes of) formulas with at most one free variable, $v . f(Y)$ is simply the code number of $Y$. This is obviously into.

All that remains to do is to extend this to a model of the Satisfaction Schema. This is done by an iterative construction which delivers an appropriate fixed-point. I will not hammer through the details here. For truth, rather than satisfaction, the details can be found in the Appendix of Priest (1991), and also Priest (2002b), §8. The argument for satisfaction is completely analogous. We set the extension of $\sigma$ at level $\alpha+1$ to be those pairs $\langle n, \# B\rangle$ where $B$ is a sentence with at most one free variable, and $n$ satisfies $B$ at level $\alpha$. At limit ordinals, we take intersections. By the usual cardinality considerations, there is a fixed point of the construction, which verifies the Satisfaction Schema.

The theory is non-trivial. All the purely arithmetic formulas behave consistently. Indeed, all formulas which are grounded - in essentially the sense of Kripke (1975) - are consistent in it.

Note also the following. We have interpreted propositions and properties syntactically. This places constraints on their properties, and especially their identity conditions. It might therefore be thought that this limits the significance of the consistency results. It does not. For anything that is consistent, given these constraints, is certainly consistent with weaker constraints - and so for less fine-grained identity conditions. 


\section{Conclusion}

Finally, let us look more closely at our paradox-generating formula, $\exists Y(v=$ $f(Y) \wedge \neg v \sigma Y)$. In the model, ' $Y$ ' ranges over (codes of) formulas with at most one free variable, and $f(Y)$, that is, $Y$, is that code. So our formula becoms simply: $\neg v \sigma v$, where $v$ ranges over monadic properties or predicates. ${ }^{10}$ To say that $v$ does not satisfy itself is, of course, the condition which delivers the Heterological Paradox. ${ }^{11}$ So just as Cantor's Paradox can seen as collapsing into Russell's, ${ }^{12}$ the Russell/Myhill/Kaplan paradox can be seen as collapsing into this. ${ }^{13}$

\section{References}

[1] Kaplan, D. (1995), 'A Problem in Possible World Semantics', ch. 4 of Modality, Morality and Belief: Essays in Honor of Ruth Barcan Marcus, Cambridge: Cambridge University Press.

[2] Kripke, S. (1975), 'Outline of a Theory of Truth', Journal of Philosophy 72: $690-716$.

[3] Myhill, J. (1958), 'Problems Arising in the Formalization of Intensional Logic', Logique et Analyse 1: 78-83.

[4] Priest, G. (1991), 'Intensional Paradoxes', Notre Dame Journal of Formal Logic 32: 193-211.

[5] Priest, G. (2002a), Beyond the Limits of Thought, 2nd edn, Oxford: Oxford University Press.

[6] Priest, G. (2002b), 'Paraconsistent Logic', pp. 287-393, Vol. 6, of D. Gabbay and F. Guenthner (eds), Handbook of Philosophical Logic, 2nd edn, Dordrecht: Kluwer Academic Publishers.

\footnotetext{
${ }^{10}$ Taking propositions or closed sentences to be degenerate cases of these.

${ }^{11}$ See Priest (2006), 1.2.

${ }^{12}$ See Priest (2002a), 9.1.

${ }^{13} \mathrm{~A}$ version of this paper was given at the conference Cardinality, Worlds, and Paradox, University of Oslo, June 2016. Thanks go to the participants of the conference for their comments.
} 
[7] Priest, G, (2006), In Contradiction, 2nd edn, Oxford: Oxford University Press.

[8] Priest, G. (2017), 'What If? The Exploration of an Idea', Australasian Journal of Logic 14, https://ojs.victoria.ac.nz/ajl/article/view/4028/3574.

[9] Russell, B. (1903), The Principles of Mathematics, Cambridge: Cambridge University Press. 


\section{University Library}

\section{- M M N E R VA A gateway to Melbourne's research publications}

Minerva Access is the Institutional Repository of The University of Melbourne

Author/s:

Priest, $\mathrm{G}$

Title:

PARADOXICAL PROPOSITIONS

Date:

2018-10-01

Citation:

Priest, G. (2018). PARADOXICAL PROPOSITIONS. PHILOSOPHICAL ISSUES, 28 (1), pp.300-307. https://doi.org/10.1111/phis. 12126.

Persistent Link:

http://hdl.handle.net/11343/284697 